\title{
Detrusor underactivity versus bladder outlet obstruction clinical and urodynamic factors
}

\author{
Jefferson Kalil ${ }^{1}$, Carlos Arturo Levi D’Ancona ${ }^{1}$ \\ ${ }^{1}$ Escola de Ciências Médicas, Universidade Estadual de Campinas - Unicamp, Campinas, SP, Brasil
}

\section{ABSTRACT}

Objectives: To evaluate the lower urinary tract symptoms, classified by the International Prostate Symptom Score (IPSS), urodynamic results (Watts Factor (WF), Bladder Contractility Index (BCI), and post void residual (PVR), in order to differentiate Detrusor Underactivity (DU) from Bladder Outlet Obstruction (BO0).

Methods: Retrospective observational study performed from 2011 to 2018 at the Hospital das Clínicas of Unicamp. Two phases were done: first, to estimate sample size, and second, to evaluate the predicted parameters. Male patients with range age from 40 to 80 years were included. Patients were divided into two groups: Group 1, without B00 and with DU; Group 2, with B00. Variables analyzed: age, comorbidities, symptoms, urodynamic data (BCI and WF) and PVR.

Results: Twenty-two patients were included in each group, with medians of 68 (Group 1) and 67.5 years old (Group 2) ( $p=0.8416)$. There was no difference for comorbidities. In relation to IPSS, medians were: 16.5 and 20.5 , respectively $(p=0.858)$. As for symptoms, there was predominance of combination of storage and voiding symptoms in the two groups $(\mathrm{p}=0.1810)$. Regarding PVR, 15 patients in Group 1 and 16 in Group 2 presented PVR $>30 \mathrm{~mL}(\mathrm{p}=0.7411)$. BCI presented median values of 75 and 755.50 for Group 1 and Group 2, respectively ( $\mathrm{p}<0.0001)$, while WF had medians of 22.42 and $73.85(\mathrm{p}<0.0001)$.

Conclusion: Isolated symptoms, classified by IPSS and PVR, could not differentiate patients with DU from those with B00, but it was possible using urodynamic data.

\section{ARTICLE INFO}

iD Jefferson Kalil

http://orcid.org/0000-0001-6624-8074

\section{Keywords:}

Urodynamics; Urinary Bladder, Underactive; Lower Urinary Tract Symptoms

Int Braz J Urol. 2020; 46: 419-24

\section{Submitted for publication:}

June 18, 2019

Accepted after revision:

January 18, 2020

Published as Ahead of Print:

February 10, 2020

\section{INTRODUCTION}

Detrusor underactivity (DU) according to the International Continence Society (ICS) includes low detrusor pressure or short detrusor contraction time, usually in combination with a low urine flow rate resulting in prolonged bladder emptying and/or failure to achieve complete bladder emptying within a normal time span (1).
This definition, although adequate, does not show the parameters that define this diagnosis: method used to measure strength, value of normality and appropriate urination time.

In general, the most prevalent symptoms in men with DU are: reduction and/or interruption of urinary flow, hesitancy, incomplete emptying sensation, palpable bladder, absence and/or reduction of sensitivity, and effort to urinate (2). Recently, si- 
milarly to what occurs with the Overactive Bladder, a new clinical syndrome has been suggested, called Underactive Bladder Syndrome, which presents the following symptoms: prolonged urination time with or without feeling of incomplete emptying, usually with hesitancy, reduced sensation of filling, and weak stream (3). Nevertheless, neither the symptoms nor the urodynamic criteria that define DU are well defined so far. This is important, since the prevalence of DU is $9 \%$ to $23 \%$ in men and $12 \%$ to $45 \%$ in women (4). Differentiating patients with DU associated with bladder outlet obstruction (B00) from those who present only bladder outlet obstruction is relevant, since the former group may not benefit from surgical treatment, while the latter will certainly do (5). Moreover, patients with DU and chronic urinary retention may develop overflow incontinence and reduced urination output, which are mild complications, in addition to severe complications, such as urinary lithiasis, recurrent urinary tract infections and renal insufficiency (6).

Regarding the diagnosis, it is necessary to use urodynamics, which is an invasive investigation. Classically, detrusor strength has been evaluated by means of pressure/flow studies. It is noteworthy that current methods used to evaluate the detrusor function are: Schafer nomogram (pressure/ flow), Bladder Contractility Index (BCI) and Watts Factor (WF) (7).

Schafer nomogram has been used to differentiate patients with DU from patients with BOO $(7,8)$. BCI is calculated using a simple formula and can be used as screening method for DU, although it may be a method that is not able to differentiate patients with DU from those with BOO $(7,9)$. Finally, WF is calculated by a mathematical formula that evaluates the power applied on the bladder per area unit, and calculates detrusor strength during an isovolumetric contraction $(10,11)$.

Considering the limitations of all mentioned methods, two methods which would have few interfering elements were selected: Bladder Contractility Index (BCI) and Watts Factor (WF) $(4,12)$.

Despite the fact that some evidence suggests symptoms and post void residual can indicate DU, the information is not well consolidated and the differentiation between DU and BOO remains a problem.
The aim of this study is to evaluate the validity of the lower urinary tract symptoms (LUTS), International Prostate Symptom Score (IPSS), BCI, $\mathrm{WF}$ and post void residual to differentiate DU from BO0.

\section{PATIENTS AND METHODS}

Comparative retrospective cross-sectional observational study performed between two groups: Group 1, patients without B00 and with diagnosis of DU, Group 2, patients with B00. Two phases were performed: the first one, in order to calculate the sample size, and the second, with the final results. Data from the study were obtained from the database of urodynamic exams performed and stored at the Urodynamic Service of HC-UNICAMP (Hospital das Clínicas of the University of Campinas), from patient's medical charts and from HC Computer System. This study was approved by the Ethics Committee in Research, number 1.644.754.

All patients were male and underwent urodynamics between 2011 and 2018. Criteria for inclusion in Group 1 were: absence of obstruction or doubt in ICS Nomogram and presence of weak detrusor in Schafer Nomogram. Inclusion criteria in Group 2 was presence of obstruction in ICS Nomogram. Exclusion criteria were: presence of neurological disease, postoperative of pelvic exenteration, bladder cancer, prostate cancer and patients who underwent prostatic surgery.

In the first phase of the study, 5\% significance level (alpha or type I error), and 80\% sample power (beta or 20\% type II error) were used for sample size calculation. The calculation was made considering the comparison of variables between the two groups. For BCI and WF, 22 and 20 patients were respectively required in each group. Therefore, the sample was enlarged in order to obtain 22 patients in each group.

Besides age and comorbidities, symptoms were analyzed through the International Prostate Symptom Score (IPSS) and classified as storage, voiding, combination of storage and voiding symptoms, and urinary incontinence, urodynamic data: post void residual (PVR) and classification of ICS and Schafer nomograms and calculation of Bladder Contractility Index and Watts Factor. 
Categorical variables were described using absolute frequency and percentage, and numerical variables were described as mean, median, standard deviation, minimum and maximum. Mann-Whitney test was used for numerical variables, Chi-square test for PVR and Fisher's exact test for symptoms, considering a 5\% significance level.

\section{RESULTS}

A total of 44 patients were included in the study, 22 in each group. The average age was 68 years and 67.5 years for Group 1 and Group 2, respectively. $(\mathrm{p}=0.8416)$.

Arterial hypertension was present in the same proportion between groups $(64 \%)(p=1.0)$, while Diabetes Mellitus appeared in 23\% of patients in Group 1 and 36\% in Group 2 ( $p=0.51$ ). Dyslipidemia was present in 27\% in Group 1 and $18 \%$ in Group 2 ( $\mathrm{p}=0.72$ ), and Acute Myocardial Infarction appeared in 23\% and 5\% patients in Groups 1 and 2, respectively ( $\mathrm{p}=0.18)$, patients without comorbidities represented 17\% in Group 1 and 18\% in Group 2 ( $p=1.0$, Fisher's exact test).
For IPSS, the following means were obtained: $16.5(\mathrm{SD}=5.76)$ for Group 1 and 20.5 $(\mathrm{SD}=6.94)$ for Group 2, without significant difference ( $\mathrm{p}=0.858$, Mann-Whitney test). For symptoms, the most prevalent category in both groups was the combination of storage and voiding symptoms with 12 patients in Group 1 and 15 patients in Group 2. However, there was not significant difference between groups ( $p=0.1810$, Fisher's exact test).

Post void residual $\geq 30 \mathrm{~mL}$ was found in 15 patients in Group 1 and 16 patients in Group 2 , without significant difference $(p=0.7411$, Chi-Square test).

BCI presented median of $75(S D=120.43)$ in Group 1, while in Group 2 median was 755.50 $(\mathrm{SD}=408.12)$, with significant difference ( $\mathrm{p}<0$, 0001, Mann-Whitney Test). For WF, Group 1 showed median of $22.42(\mathrm{SD}=14.88)$ and Group 2 median of $73.85(\mathrm{SD}=39.86)$, also with significant difference ( $p<0.0001$, Mann-Whitney test). On the other hand, age and post void residual did not show statistically difference $(\mathrm{p}=0.8416$ and $\mathrm{p}=0.5327$, respectively) (Tables 1 and 2).

Table 1 - Frequency of urodynamic data: measure of detrusor strength by Schafer Nomogram; values of BCI and WF, considering cut-off point values for Detrusor Underactivity.

\begin{tabular}{|c|c|c|}
\hline VARIABLES & GROUP $1-n(\%)$ & GROUP $2-n(\%)$ \\
\hline \multicolumn{3}{|l|}{ Schafer } \\
\hline Very weak & $3(13.64 \%)$ & $1(4.55 \%)$ \\
\hline Weak & $18(81.82 \%)$ & $4(18.18 \%)$ \\
\hline Normal (-) & $1(4.55 \%)$ & - \\
\hline Normal (+) & - & $12(54.55 \%)$ \\
\hline Strong & - & $5(22.73 \%)$ \\
\hline \multicolumn{3}{|l|}{ BCI } \\
\hline$\leq 100$ & $17(77.27 \%)$ & $2(9.09 \%)$ \\
\hline$>100$ & $5(22.73 \%)$ & $20(90.91 \%)$ \\
\hline \multicolumn{3}{|l|}{ WF } \\
\hline$\leq 7$ & $7(31.82 \%)$ & $2(9.09 \%)$ \\
\hline$>7$ & 15 (68.18\%) & $20(90.91 \%)$ \\
\hline
\end{tabular}


Table 2 - Descriptive and comparative analysis between groups: age, urodynamic data and post void residual; mean, median, standard deviation, minimum, maximum, and confidence intervals were described. There was a significant difference between groups for $\mathrm{BCl}$ and WF ( $\mathrm{p}<0.0001$; Mann-Whitney test).

\begin{tabular}{lcccccccc}
\hline \multirow{2}{*}{ VARIABLES } & \multicolumn{2}{c}{ Age (years) } & \multicolumn{2}{c}{ BCI } & \multicolumn{2}{c}{ WF } & \multicolumn{2}{c}{ PVR (mL) } \\
\cline { 2 - 8 } Nroup 1 & Group 2 & Group 1 & Group 2 & Group 1 & Group 2 & Group 1 & Group 2 \\
\hline N & 22 & 22 & 22 & 22 & 22 & 22 & 22 & 22 \\
Mean & 68.23 & 67.5 & 125.41 & 782.82 & 21.3 & 73.33 & 99.36 & 134.77 \\
Standard Deviation & 10.85 & 9.29 & 120.43 & 408.12 & 14.88 & 39.86 & 93.43 & 146.18 \\
Minimum & 50 & 45 & 43 & 68 & 1.66 & 2.73 & 0 & 0 \\
Maximum & 86 & 83 & 528 & 1677 & 51.37 & 182 & 350 & 620 \\
$\mathbf{p}$ & 0.8416 & & $<0.0001$ & & $<0.0001$ & & 0.5327 & \\
\hline
\end{tabular}

$\mathbf{B C I}=$ Bladder Contractility Index; $\mathbf{W F}=$ Watts Factor; $\mathbf{P V R}=$ Post Void Residual

\section{DISCUSSION}

In a recent study comparing patients with DU and B00, age was not different between groups, as in our study (13). However, it is worth noting that literature shows that DU is more common in the elderly, as demonstrated here, with median age of 68 years $(4,13-15)$.

Regarding comorbidities, a study comparing these two diseases also showed no difference between groups for hypertension, DM and other diseases, as demonstrated in this study (16). However, it is interesting to note that duration of hypertension (more than 10 years) and DM (more than 6 years) resulted in detrusor contractility changes (13). It is relevant because DM is a disease that leads to nerve damage and deposition of collagen and extracellular matrix in the detrusor, leading to changes on its function $(17,18)$.

IPSS score, classically described to evaluate the severity of prostate-related symptoms, is presented as a numerical index able to classify symptoms into categories such as mild, moderate or severe. As we have seen, Group 1 did not present statistically difference from Group 2, such as demonstrated by other authors who compared groups of patients with DU and BOO using IPSS score (14). Meanwhile, another study showed a correlation between DU and IPSS, with an association of IPSS between 20-23 and DU (13). Therefore, there is not consensus on validity of IPSS score and its cut-off to DU.

Regarding symptoms, their combination was predominant in both groups in this study, however, an interesting fact is that no patient had only storage symptoms in Group 2, but this information was not significant. Corroborating this information, another study comparing men with DU and BOO showed no difference in symptoms between groups (19). Thus, it seems that the category of symptoms alone cannot differentiate these diagnoses, but it needs to be investigated in further studies.

For BCI, literature places the cut-off value as 100. According to these criteria, $\mathrm{BCI}<100$ corresponds to weak detrusor, between 100-150, normal detrusor, and $>150$, strong detrusor (12). As observed in Table 2, there is a statistically difference between the groups for BCI, showing that this could be a good parameter, in opposition to what has been presented in other articles that $\mathrm{BCI}$ is not able to differentiate patients with DU from those with $\mathrm{BOO}(8,20)$.

The second parameter, Watts Factor, also presented statistical difference between the groups, so it could be a good indicator as well. For WF, li- 
terature suggests the value of $7 \mathrm{~W} / \mathrm{m} 2$ as a limit of normality (10). However, although this cut-off has been suggested to the diagnosis of DU, it could be a parameter to differentiate patients with DU from those with BO0 (20).

Taking into account the results of our study we suggest the cut-offs of BCI and WF to classify DU and BOO could vary from the classical cut-offs of $7 \mathrm{~W} / \mathrm{m} 2$ to $\mathrm{WF}$ and 100 to BCI. However, at present, this cannot be accepted as truth, but should be assessed in future studies.

One study compared Schafer Nomogram, BCI and WF, and showed that only Schafer Nomogram would be able to identify B00, however, we could observe from the data presented that both $\mathrm{BCI}$ and WF were able to differentiate DU from BOO (8).

In another study, there was a correlation among BCI, WF and B00, inferring that as the degree of bladder outlet obstruction increases, BCI and WF values would increase proportionally. In this line, the authors of that study question the cut-offs used in the diagnosis of DU, suggesting that low values $(\mathrm{BCI}<100$ and $\mathrm{WF}<7)$ would be present since there is not $\mathrm{BOO}$ and the detrusor muscle is not required to increase its strength in order to compensate resistance. However, when there is DU, there is no increase in detrusor pressure, since there is detrusor failure. Nevertheless, as the study itself presented, there was statistical difference for BCI and WF when compared to Bladder Outlet Obstruction. They questioned both the DU diagnosis criteria and the possible parameters that would compare DU with B00, but affirmed that these were initial conclusions and needed to be validated (14). One factor to be taken into account is that this compensatory increase could reflect the initial phase of the disease, with the detrusor muscle is still strong, and the second moment, in the evolution of the disease, when it would lose its strength (21). However, in the current studies, there is not reference on the possible DU evolution time and this should be considered in future studies.

One study demonstrated that patients with DU presented high PVR when compared to the control group. In this study, for the diagnosis of DU, the cut-off was $147 \mathrm{~mL}$, with sensitivity and specificity of $60.16 \%$ and $72.97 \%$, respectively, furthermore, these authors showed that PVR was an independent predictor for DU (13). Although more data suggest that residual volume $>40 \%$ maximum cystometric capacity would be a strong indicator of DU, it is not a consensus (4). Here, PVR did not show statistical difference between groups, indicating that PVR alone would not be able to differentiate diagnoses, as demonstrated in other studies, which also did not show significant difference between Obstructed and Non-obstructed $(14,19)$. Thus, although PVR can be calculated using a non-invasive method, such as abdominal ultrasonography, it cannot yet be considered a reliable diagnosis factor, requiring further studies.

The limitations of this study were the small number of patients, the quality of data collected, some missing data, because it is a retrospective study, and the need to classify the symptoms into categories, rather than considering each one separately, because the data collected was registered in categories of symptoms.

\section{CONCLUSIONS}

It is possible to conclude that the lower urinary tract symptoms, the International Prostate Symptom Score and post void residual did not show difference between Detrusor Underactivity and Bladder Outlet Obstruction in this study, in contrast, Watts Factor and Bladder Contractility Index were relevant tools in this differentiation.

\section{CONFLICT OF INTEREST}

None declared.

\section{REFERENCES}

1. D'Ancona C, Haylen B, Oelke M, Abranches-Monteiro L, Arnold E, Goldman H, et al. The International Continence Society (ICS) report on the terminology for adult male lower urinary tract and pelvic floor symptoms and dysfunction. Neurourol Urodyn. 2019;38:433-77.

2. Gammie A, Kaper M, Dorrepaal C, Kos T, Abrams P. Signs and Symptoms of Detrusor Underactivity: An Analysis of Clinical Presentation and Urodynamic Tests From a Large Group of Patients Undergoing Pressure Flow Studies. Eur Urol. 2016;69:361-9. 
3. Chapple CR, Osman NI, Birder L, van Koeveringe GA, Oelke M, Nitti VW, et al. The underactive bladder: a new clinical concept? Eur Urol. 2015;68:351-3.

4. Osman NI, Chapple CR, Abrams P, Dmochowski R, Haab $F$, Nitti $V$, et al. Detrusor underactivity and the underactive bladder: a new clinical entity? A review of current terminology, definitions, epidemiology, aetiology, and diagnosis. Eur Urol. 2014;65:389-98.

5. Rademakers KL, van Koeveringe GA, Oelke M. Detrusor underactivity in men with lower urinary tract symptoms/ benign prostatic obstruction: characterization and potential impact on indications for surgical treatment of the prostate. Curr Opin Urol. 2016;26:3-10.

6. Abrams P, Cardozo L, Fall M, Griffiths D, Rosier P, Ulmsten $\mathrm{U}$, et al. The standardisation of terminology of lower urinary tract function: report from the Standardisation Sub-committee of the International Continence Society. Neurourol Urodyn. 2002;21:167-78.

7. Saito M, Yokoi K, Ohmura M, Kondo A. Effects of partial outflow obstruction on bladder contractility and blood flow to the detrusor: comparison between mild and severe obstruction. Urol Int. 1997;59:226-30.

8. Chang $\mathrm{YH}$, Siu JJ, Hsiao PJ, Chang $\mathrm{CH}$, Chou EC. Review of underactive bladder. J Formos Med Assoc. 2018;117:178-184.

9. Schäfer W. Analysis of bladder-outlet function with the linearized passive urethral resistance relation, linPURR, and a disease-specific approach for grading obstruction: from complex to simple. World J Urol. 1995;13:47-58.

10. Abrams P. Bladder outlet obstruction index, bladder contractility index and bladder voiding efficiency: three simple indices to define bladder voiding function. BJU Int. 1999;84:14-5.

11. Lecamwasam HS, Yalla SV, Cravalho EG, Sullivan MP. The maximum watts factor as a measure of detrusor contractility independent of outlet resistance. Neurourol Urodyn. 1998;17:621-35.

12. Derek J. Griffiths: Assessment of detrusor contraction strength or contractility. Neurourol Urodyn. 1991; 10: 1-18.

13. Luo F, Sun HH, Su YH, Zhang ZH, Wang YS, Zhao Z, et al. Assessment of noninvasive predictors of bladder detrusor underactivity in BPH/LUTs patients. Int Urol Nephrol. 2017;49:787-92.
14. Oelke $\mathrm{M}$, Rademakers $\mathrm{KL}$, van Koeveringe GA. Detrusor contraction power parameters $(\mathrm{BCl}$ and $\mathrm{W}$ max) rise with increasing bladder outlet obstruction grade in men with lower urinary tract symptoms: results from a urodynamic database analysis. World J Urol. 2014;32:1177-83.

15. Madersbacher S, Pycha A, Schatzl G, Mian C, Klingler CH, Marberger $M$. The aging lower urinary tract: a comparative urodynamic study of men and women. Urology. 1998;51:206-12.

16. Brierly RD, Hindley RG, McLarty E, Harding DM, Thomas PJ. A prospective controlled quantitative study of ultrastructural changes in the underactive detrusor. J Urol. 2003;169:1374-8.

17. Lee WC, Wu HP, Tai TY, Liu SP, Chen J, Yu HJ. Effects of diabetes on female voiding behavior. J Urol. 2004;172:989-92.

18. Miyazato M, Yoshimura N, Chancellor MB. The other bladder syndrome: underactive bladder. Rev Urol. 2013;15:11-22.

19. Jeong SJ, Kim HJ, Lee YJ, Lee JK, Lee BK, Choo YM, et al. Prevalence and Clinical Features of Detrusor Underactivity among Elderly with Lower Urinary Tract Symptoms: A Comparison between Men and Women. Korean J Urol. 2012;53:342-8

20. van Koeveringe GA, Vahabi B, Andersson KE, KirschnerHerrmans R, Oelke M. Detrusor underactivity: a plea for new approaches to a common bladder dysfunction. Neurourol Urodyn. 2011;30:723-8.

21. Jiang $\mathrm{YH}$, Lin VC, Liao $\mathrm{CH}$, Kuo HC. International Prostatic Symptom Score-voiding/storage subscore ratio in association with total prostatic volume and maximum flow rate is diagnostic of bladder outlet-related lower urinary tract dysfunction in men with lower urinary tract symptoms. PLoS One. 2013;8:e59176.

Correspondence address: Jefferson Kalil, MD

Escola de Ciências Médicas, Universidade Estadual de Campinas - Unicamp, Rua Tessália Vieira de Camargo, 126, Cidade Universitária Zeferino Vaz Campinas, SP, 13083-887, Brasil Telefone: + 5519 3521-8863 E-mail: jeffkalil@hotmail.com 